\title{
LAS PARASITOSIS HEPATOBILIARES EN EL ECUADOR (*)
}

Drs.: Luis A. León y Renato León C., Quito

El estudio de las parasito'sis del hígado y de las vías biliares es áe enorme importancia desde el punto de vista clínica e histopatológico, como también por su gran frecuencia y gravedad. Su diagnóstico, si bien se puede establecer en muchos casos merced a los datos clínicos, de laboratorio y a los modernos métodos de exploración, pero no en raras ocasiones se descubren estas parasitosis en las intervenciones quirúrgicas y en las mesas die autopsia.

El doctor Tomás Quevedo Gómez, (**) con el título de "parasitosis $\mathrm{He}$ partobiliares en Colombia" publicó un interesante trabajo de recopilación, en el cual nos dio a conocer: dos casos de quistes hidatídicos, tres de estrongiloidiasis, catorce casos de fascioliasis veinte $\mathrm{y}$ dos ya publicardos y diez $\mathrm{y}$ siete nuevos de ascaridiosis hepatobiliares. Como podemos apreciar por estos datos, el doctor Quevedo Gómez

\footnotetext{
*) Tomado de GACETA MEDICA DE CARACAS, Año LXXXVII, Nos. 7 8-9, de Julio-Septiembre de 1979, pp. 271-283. Caracas - Venezuela.

( *) QUEVEDO GOMEZ, T. Parasitosis Hepatobiliares en Colombia. Revista "El Médico de Colombia y Ecuador", 3 (3): 411-,1958.
}

nos informa tan sólo de parasitosis por helmintos. En la magistral obra Parasitología Clínica por los ilustres Profesores, Antonio Atias y Amador Neghme (***), que acaban de publicar, en la parte correspondiente al capítulo Parasitología Topográfica se consideran las siguientes parasitosis del higado:

1) Afecciones difusas del parenquima: Malaria, Kala-azar, Enfermedad de Chagas y Toxoplasmosis.

2) Afecciones localizadas en el $\mathbf{P a}$ renquima: a). Abscesos: amibianos y ascaridiosis; b) Granulomas: larva migrante visceral y granulomas por huevos die helmintos (capitariasis, esquistosomiasis y escariasis) (c. Quistes: hidatidosis multilocular y cisticercosis.

3) Afecciones fibróticas del hígado: Esquistosomiasis y fascioliasis.

4) Afecciones obstructivas: a) de la vía biliar: fascioliasis, hidatidosis y ascariasis. b) de la vía hemática: esquistosomiasis e hidatidosis.

(***) ATIAS, A. Y NEGHME, A. Paresitología Clínica, po. 339-344. InterMédica, Editorial, Buenos Aires, 1979 
En el Tratado de Gastroenterología por el doctor Juan Nasio (****), encontramos algunos capítulos sobre este tema, en los cuales se han estudiado por separado los siguientes: Parasitación extensa del hígado por quistes hidatídicos, todos periféricos; Parasitismo por Fasciola hépatica; Parásitos en la cirrosis diel hígaido; parásitos biliares, etc.

En el Ecuador, de acuerdo con la literatura médica nacional y nuestras propias observaciones, las parasitosis del hígado y de las vías biliares son enfermedades más numerosas y dignas de darse a conocer como un valioso aporte a la Parasitología y a la Gastroenterología de Latinoamérica. En forma sinóptica daremos a conocer las paras tosis hepatobiliares observadas en nuestro país, siguiendo la sistemática de los agentes etiológicos. El estudio de estas parasitosis de acuerdo con la base histopatológica, si bien da un concepto claro de las lesiones que ocasionan los parásitos en dichos órganos, en cambio no permite describirlas con sujeción a la taxonomía de dichos agentes. En esta revisión de las parasitosis, prescindiremos de los aspectos morfológico, biológico, patogénico die los diferentes parásitos, asi como también del diagnóstico y tratamiento, que sería uno de tantos temas a tratarse con detalles.

\footnotetext{
(****) NASIO, J. Tratado de Gastroenterología. Tomo III, pp. 324, 385, 409 y 739. Salvat Editores, S. A. Barcelona, Madrid. 1961.
}

\section{PROTOZOASIS}

AMEBIASIS Las formas vegetativas de la Entamoeba histolytica Schaudiin, 1903, en sus invasiones al hígado provoca: hepatitis, abscesos y en ocasiones, formas tumorales y cirróticas del hígado. Sin embargo de que los grandies tropicalistas, como Sir Leonardo Rogers, Sir Patrick Manson, Sir Philip Manson-Bahr, Richard Strong, Ch. Joyeux, George Cheever Shattuck, Everard Napier, Julio Guiart y Carlos Garin, etc. y los célebres parasitólogos norteamericanos Craig y Faust, consideran en sus obras las hepatitis amibianas, o hepatitis amibiana no supuradla o hepatitis tropical, nuestros médicos poco o nadla hablan de esta complicación, pese a que es sumamente frecuente en nuestro medio $\mathrm{y}$ que requiere tratamiento oportuno; tan sólo se han preocupado de ella: el mallogrado y distinguido clínico doctor Juan Tanca Marengo, (1) quien trató de la hepatitis aguda congestiva y de la hepatitis crónica congestiva al considerar las diferentes formas clínicas de esta enfermedaid; el doctor Amadeo Moneira (2) también nos ofreció un valioso estudio sobre la clínica y el tratamiento de la hepatitis amibiana; igualmente, Ricardo Barzallo (3) abordó el estudio de la hepatitis amibiana del lóbulo izquierdo (formas tórpidas), y finalmente, el doctor Leoncio Cordero Jaramillo (4) señaló algunas modificaciones encontradas dlesde el punto de vista histopatológico de la amibiasis.

En cuanto al absceso hepático o absceso tropical del hígado, el célebre 
historiador de la Medicina Latinoamericana, doctor Arístides Moll (5), nos refiere en su obra que el cirujano italiano Anacleto Cervini por el año de 1883 introdujo en el Ecuador nuevos métodos operatorios, incluyendo la apiertura del albsiceso hepático; por los años de 1896 a 1897, el médico, ciruja. no y oftalmólogo, doctor J. M. Troya (6) dejó las indlicaciones del tratamiento quirúngico de las "postemas" o "apostemas" del hígado; también a fines diel siglo XIX el doctor Juan Destruge (7) publicó un interesante estudio sobre abcesos hepáticos; a principios del siglo XX, M. García nos dabló en forma detenida sobre el tratamiento de esta complicación de igual manera que el sabio médico francés, Paul Rivet (1901).

Por el año de 1929 el doctor Julio Enrique Paredes (8) publicó en Anales de la Universidad Central su artícula "Forma de Pseudo-Neoplásica en la Hepatitis Amibiana", y el doctor Juan Tanca Marengo (1) en su clasificación de la amibiasis hepática también consideró las formas tumorales Las formas nodular hipertrófica con cirrosis, observada en Europa por Achard, Menetrier y Brodín, han sido señaladas por algunos médicos nacionales en necropsias de pacientes que han tenido antecedentes amibianos.

Las amibiasis del hígado son, pues, $\tan$ frecuentes y graves en nuestro medio que nos ha llevado a realizar un estudio detenido sobre la historia de esta complicación (9).

MALARIA. Desde la época de Hipócrates la hepatomegalia ya fue considera como una manifestación clí- nica del paludismo, figurando entre sus múltiples complicaciones la fiebre biliosa hemoglobinúrica, estudiada ampliamente por Roux, Noch y Pitaluga y que el proceso mórbido compromete gravemente al hígado, a igual que al cerebro, a las meninges y al bazo. Por Shortt, Garnham, Fairley y otros investigadores sabemos, ahora, que los ciclos preeritrocítico y exoeritrocítico del hematozoario de Laverán se realizan en las células hepáticas provocandio profundas alteraciones de la glándula En la caquexi a palúdica, ocasionada principalmente por ell Plasmodium falciparum, el hígado y el bazo, bien se mantienen grandes y endurecidos, o se atrofian.

En el Ecuador el paludismo llegó a ser erradicado de sus valles interandinos, pero en el Litoral y en la Región Orientali todlavía const'tuye un grave problema médico y sanitario.

COCCIDIOSIS. Aunque esta parasitosis de localización hepática es muy rara, en el Ecuador, Ieón y Guerrero (10) en 1959 reportaron, por necropsia, un caso provocado por una especie del género Eimeria. La paciente acusaba episodios dolorosos del epicondrio derecho, acompañados de alza térmica, de náuseas intensas, a veces de diarreas amarillentas; a su ingreso al hospital "Eugenio Espejo" de Quito, presentó subictericia, piel sudorosa, dolores musculares y óseos, hígado grandie, duro y dioloroso, sobre todo a nivel del lóbulo izquierdo; el examen de sangre acusó: 4.370 .000 hematíes $\mathrm{x}$ mmc., 32.000 leucocitos, jóvenes el $2 \%$, cayados el $44 \%$, segmentados el $50 \%$, linfocitos el $3 \%$ y monocitos el $1 \%$; 
presencia de formas anulares de Plasmodium falciparum, velocidad de sedimentación: 1ra. hora $40 \mathrm{~mm}$. y 2 da. hora, $80 \mathrm{~mm}$. El examen de orina reveló 37 miligrs. de albúmina, 10 a 15 hematíes por campo. Pese al tratamiento de la infecc ón palúdica la paciente falleció al $16^{\circ}$ día. En ell protocolo de autopsia se encontró un foco purulante circunscrito a la trascavidad de los epiplones, cuyo pus era de color amarillento, grumoso, espeso y mal oliente; el hígaido de 1.440 gramos de peso presentaba adherencias con el lóbulo de Spiegel, y en la parte correspondiente al lóbulo izqui erdo se encontró porciones desflecadas húmedas y brillantes. Al corte se estableció que la mayor parte de aquel lóbulo estaba remplazado por un material pardo amarillento, friable; la mayor parte de las serosas parietales presentaban procesos de serositis fibrinosa. El examen histopatológico del parenquima hepático que rodeaba al foco purulento puso de manifiesto la presencia de abundantes coccidias en proceso de multiplicación y numerosos oocistes. Consideramos que en América es uno de los poicos casos de coccidiosis hepática humana.

TOXOPLASMOSIS. Con relativa frecuencia estamos diagnosticando en nuestro laboratorio casos de esta enfermedaid en sus formas congénitas y adquiridas. En estas últimas formas el Toxoplasma gondii se localiza en el tejido retículoendotelial del paciente provocanido varias formas clínicas, tales como meningoencefalitis, coroidoretinitis, miocarditis, neumonía atípica y cuadros patológicos de infeccio- nes generalizadas que se semejan al tifus exantemático y en cuyas formas los parásitos se localizan también en el hígado, bazo, suprarrenales, ri ñones, ganglios linfáticos, etc. Por tanto, siempre encontraremos en estos procesos de infección generalizaidia transtornos del hígado. En la toxoplaismosis congénita, además del sindrome de Sabin (hidracefalia, coroidoretinitis y convulsiones) existe hepato y esplenomegalia.

LEISHIMANIASIS VISCERAL O KALA-AZAR. Después de que clinicamente los doctores Juan Federico Heinert (11) y Alfedo Valenzuela diagnosticaron en Guayaquil esta flagelosis, en 1949 tuvimos ocasión de comprobar clínica y parasitologicamente la existencia en el país de dicha leishmaniasis. Posteriormente, en un corte histopatológico del hígado practicado en el laboratorio de Patología del Hospital "Eugenio Espejo" descubrimos Leishmanias donovani en dicho corte, por desgracia no se pudo conocer la procedencia del paciente; luego más tarde en un niño nativo de la provincia de Esmeraldas, pudimos diagnosticar clínica y por punción biopsia del hígado el tercer caso de kala-azar.

La leishmaniasis visceral, como es sabido, es una enfermedaid que ataca a muchas partes del organismo, pero son las localizaciones en las células del hígado (células de Kunoffer) las de mayor importancia y gravedald, sobre todo en el kala-azar infantil.

ENFERMEDAD DE CHAGAS. Es una flagelosis bastante extendida en el Litoral ecuatoriano, registrándose 
en forma esporádica en las provincias australes del país, y, posiblemente, también en el Oriente, en vista de unos pocos casos con miocarditis chagásica procedentes de esta Región. El hecho de que nuestra literatura médica cuente con más de ochenta publicaciones, nos está demostrando que esta enfermeidad, en forma endémica o de brotes epidémicos, es diagnosticada entre nosotros con relativa frecuencia. Puesto que en esta dolencia tropical existe el ataque de los Trypanosomas cruzi a muchos órganos del paciente, el hígado se compromete en la casi totalidad de los casos. En las infecciones agudas, especialmente de los niños, hay hepatomegalia y microscopicamente se descubren zonas de infartos, aumento considerable de las células de Kupffer y die los macrófagos, atrofia de las células parenquimatosas, etc. En las formas subaguidas y especialmente en las crónicas se observa infiltración de los especios porta y luego esclerosis periportal, cirrosis venosa o biliar, etc.

BALANTIDIOSIS. En 1967 el doc tor Jorge Luis Auz (12) presentó un caso de abceso hepático por Balantidium coli en un sujeto de 48 años de edad, que acusaba diarrea, pérdida de peso, astenia y posteriormente, un fuerte dolor punzante localizado en la parte superior del abidomen, siendo el dolor más intenso en el hipocondrio derecho; simultáneamente comenzó a presentar escalofríos solemnes, seguidos de fiebre alta, de malestar general. Los exámenes de sangre revelaron: 18.000 leucocitos x mmc., con el $97 \%$ de neutrófilos y el $3 \%$ de linfo- citos; $3.300 .000 \times$ mmc. de hematíes; el examen radiográfico puso de manifiesto elevación de la cúpula diafragmática derecha, suborramiento del seno costofrénico correspondiente; la imagen era de un hígado agrandado. La punción en la zona cutánea de hiperalgesia dio salida a un pus achocolatado; se procede a la laparatomía y se descubre un absceso en la cara anterior del lóbulo izquierdo del hígado y otro más grandie en el lóbulo derecho; se drena y se hace un lavado con emetina. El examen microscópico del pus da la presencia de Balantidium coli. El examen coprológico "no demuestra la preisencia de balantidios". El paciente fue traitado y curado con tetraciclinas, sulfato de aminosidina. Quizá, es el primer caso de absceso hepático por Balantidium coli reportado en Parasitología Humana. Recordemos que Bowman y otros investigadores han señalado la invasión a los ganglios linfáticos del mesenter o, a partir del intestino grueso, donde el parásito produce úlceras profundias y abscesos en la submucosa.

\section{HELMINTIASIS}

CISTICERCOSIS. Constituye un grave problema médico y sanitario del país debido a su gran frecuenclia, sobre todo por sus localizaciones cerebrales. En los casos de cisticercosis generalizada no es raro descubrirlos en el hígado. La bibliografía ecuatoriana sobre cisticercosis cerebral es abundante y ha constituído tema oficial de los congresos médicos nacionales; hemos oibservadio, además de sus locali- 
zaciones en el cerebro, cerebelo y meninges, localizaciones oculares, pulmonares y subcutáneas. El doctor Luis Espinosa Tamayo, (13) después de comentar las cinco observaciones del Dr. Alfredo Valenzuela sobre cisticercosis, dilio a conocer un caso de localización hepática; en 1926 el doctor Juan Federico Heinert (14), en un caso de cisticercosis generalizaida, muerto a conseicuencia de esta parasitosis, en la autopsia descubrió también cisticercos en el hígado; el mismo ilustre médico guayaquileño (15) en 1932 relató de otro caso en estos términos: "J. C. como afectado de cisticercosis generallzadla, especialmente del sistema muscular, pero tan intensamente parasitado y complicado de parasitosis visceral, especialmente en el corazón y en el hígado" y refirió que por entonces en su sala del Hospital (Luis Vernaza), sobre un total die cuarenta enfermos, tenía tres atacados de cisticercosis, lo cual implicaba una estadística alarmante. Largo sería citar otros estudios ecuatorianos de cisticercosis generalizada, con localizaciones hepáticas.

\section{HIDATIDOSIS. Al Primer Con-} greso Médico Ecuatoriano celebrado en 1915, los doctores Miguel Alcívar y Alfredo Valenzuela (16) presentaron el primer caso comprobado de quiste hidatídico del hígado en un ciudadano alemán, quien había poco antes residido en la Argentina; en el Registro Oficial (IV:742) del 23 de marzo de 1923 , figuran 30 defunciones por quistes hidatídicos del hígado (?); en 1926 el doctor Luis G. Dávila (17) reportó en esta ciudad un caso autóctiono de quiste hidatídico del hígado observado en autopsia. En 1948 el doctor Rafael Terán C. (18) escribió la historia clínica de un caso de esta helmintíasis operado por el doctor Aurelio Ordó$\tilde{n} \bumpeq z$, de un quiste hepático calcificado; En 1954 el doctor Luis Cueva Sotomayor diagnosticó en una intervención quirúrgica de una paciente también quiste hidatílico; igual informac ón hemos tenido de parte del doctor Alberto Bustamante de hallazgo de un quiste hidatíco del hígado en una operación quirúrgica. En 1963 los doctores Leoncio Cordero Jaramillo y Timoleón Carrera Cobos (19), mediante autopsia, descubrieron y estudiaron un caso autóctono de hidatidosis hepática; en 1965, Fortunato Zerega Pédola (20), en autopsia practicada en Guayaquil descubrieron y describieron un caso de hidatidosis hepática. Por último, los doctores Carlos Esteves Saderman, Jasé Daniel Rodríguez y Gaetano Leone (21), en 1968 presentaron a las X Jornadas Médicas, Capítulo de Guayaquil, de PAMA, un estudio de: "exéresis de quiste hidatídico unilocular" del hígadlo en un paciente nativo de Guayaquil. A esta casuística debemos añadir el caso de quiste hidatídico del hígado operado por el doctor R. Insúa en un libanés residente en Guayaquil.

En resumen, se han reportado en el país 9 casos ide hidatidosis hepática, de los cuales 7 han sido autóctonos y 2 en ciudadanos extranjeros; hemos prescindido de los 30 casos que constan en el Registro Civil, por considerar un dato erróneo, o talvez lo confundieron con cisticercosis. En el cam- 
po de la Medicina Veterinaria, el Dr. Julio Bruno Moggia no sha ofrecido un interesante estudio sobre la inciden$c$ a de la hidatidosis en el ganado que se sacrificó en la ciudlad de Guayaquil.

FASCIOLIASIS O DISTOMATOSIS HEPATICA. Con ell antecedente de que esta hemintíasis es sumamente frecuente en el ganado bovino y lanar del país y sobre todo de la Sierra y que ha sido estudiada desde fines del siglo XIX, era de suponex que en $\mathrm{Pa}$ tología humana esta helmintíasis existía también y que, qui zá, estaba pasando desapercibida, y fue tan sólo en 1971, que uno de nosotros, (22) con la colaboración del doctor Gonzalo Abald dio a conocer su existencia en el lhombre; se había hasta entonces recogido 5 observaciones: 2 casos por examen coprológico, un caso por examen die la bilis, un caso por expulsión del parásito después de un tratamiento antihelmíntico, y un caso por hallazgo por autopsia en las vías biliares; por la misma época el doctor Max Ontaneda Pólit y el Dr. Jaime Rivedeneira publicaron otra observación. Posteriormente hemos recogido cuatro observaciones más: en un niño de esta ciudad, que encontrándose en los Estados Unidos fue de urgencia operado de los conductios biliares a consecuendia de un cól co, descubriéndose en dicha intervensión quirúrgica un ejemplar de Distoma hepático; un segundo caso, que por presentar una alta eisinofilia y graves trastornos del hígado recurrió a los Estados Unidos para su diagnóstico y tratamiento, que por desgrac'a no fue establecida la naturaleza de su enfermedad y que al re- tornar a Quito, se le diagnosticó distomatosis en nuestro laboratorio, y se le sometió al tratamiento correspondiente, con el cual consiguió curarse totalmente; un tercer caso procedente de la ciudad de Ibarra, que por presentar alta eosinofilia fue tamblién env ado a nuestro laboratorio para su diagnosticó, el mismo que fue positivo para distomaitosis hepática, y el cuarto caso observado por el doctor Ernesto Gándara en una intervención quirúrgica de las vías biliares.

A estas diez olbservcaiones tenemos que añadi $r$ otras diez de un brote, con toda probaibilidad, de idistomatosis hepática registrada en el personal de una comunidad religiosa de esta Capital: hubo el antecedente de que todas las enfermas, en un paseo campestre consumienon berros en abundancia, aproximadiamente al mes y medio todas ellas comenzaron a presentar alta temperatura, mal estar general, diarrea, dolor de la región hepática y a los exámenes de laboratorio, leucocitosis con alta eosinofilia; aunque los exámenes de las heces fueron negativos para huevos del Distroma hepático, realizados por los métodos de concetración y durante toda la enfermedad, se procedió al tratamiento con dosis suficientes de Bithionol, gracias al cual todas las pacientes recuperaron totalmente la salud, corrigiéndose la leucocitosis y la gran eosinofilia. El estudio y descripción de este brote en la comunidad, será objeto de una poster or publicación con la colaboración de la médica de la comunidad religiosa, Dra. Yolanda Castro Jaramillo. 
PARAGONIMIASIS. Esta helmintíasis reportada en el país por primera vez en 1921 por doctor Juan Federico Heinert, (23) constituye, ahora, un grave problema médico y sanitario, tanto por la gravedad de sus síntomas como por su presencia en el Litoral y Región Oriental del país; varios centenares de casos han sido diagnosticados y en su mayor parte tratados. Además de las localizaciones pulmonares y algunos casos con complicaciones pleurales, en nuestro medio se han reportado locallizaciones del parásito en la piel y casos con manifestaciones cerebrales, y varios de estos casos han presentado también alteraciones hepáticas, ya que es sabido que el Paragonimus se localiza y atac'a también la viscera hépatica. La literatura ecuatoriana sobre esta trematodiasis comprende más de 40 publicaciones; nos limitaremos a citar tan sólo dos de ellas. (24 y 25).

OPISTORCHIASIS. El agente etiológico Opisthorichis (Amphimerus) guayaquilensis fue descubierto en el país por los doctores Luis Fernando Gómez Lince, José D. Roidríguez Juan Anton o Montalván (26) en 1947; el doctor Adolfo Varras Calderón

reportó posteriormente un caso, por examen de las haces con presencia de pequeños huevos operculados, de una paciente, domiciliada en Guayaquil, que adolecía de colecistitis aguda con ictericia.

Hemos observado, tanto en Guayaquil como en Quito, por examen de las heces en pacientes procedentes del Asia, que vinieron al país después de la segunda guerra mundial, pacientes atacados de clonorchiasis. En Guayaquil la reportó el doctor Julio Alvarez Crespo y en esta ciudad, el doctor Martín Rosental y los AA. y sabemos que este pequeño trematodo produce hiperplasis de las paredes de los conductos billiares y a veces adenocarcinomas del hígado.

Al tratar de las enfermedades por trematodos, aquí conviene aclarar que no se ha descubierto todavía casos comprobados y autóctonos de schistosomiasis intestinal, ni hepática. Por error, quizá, de información, los doctores Diego Ramírez, O. Jervis, Carlos Villamar, Amedeo Freire y A. Orellana, en una comunicación al IV Congreso Médico Nacional (Actas, T. I, p. 271, 1952), al considerar los parásitos intestinales más comunes en nuestro medio, sosituvieron: "schistosomiasis mansoni muy raros en nuesitro medio"; pues, quienes hemos investigado por más de 40 años las helmintíasis humanas en casi todo ell país no hemos descubierto hasta ahora casos autóctonos de schistosomiasis, pero si casos de schistosomiasis tanto intestinal como vesical procedentes del Africa; existe sí el peligro de que tarde o temprano tenga el país la fatalidad de incorporar esta terrible parasitosis a la partología nacional, en vista de que contamos con planorbis potencialmenmente huéspedes intermediarios del parásito.

ASCARIDIOSIS. Dadla la abundanclia de este vermis en el país y ante su carácter errático, hemos oído de partie die algunos cirujanos sobre el hallazgo diei Ascaris lumbricoides en las vías biliares de pacientes opera- 
dos. Aparte de estas informaciones, el doctor Germán Leone Di Vanna (28) reportó en 1957, 5 observaciones de "Granulomas y abscesos del hígado producido por Ascaris lumbricoides erráticos", correspondliendo 3 casos al material de autopsias, uno diagnosticado por punción biopsia y el restante, por laparatomía. El doctor Germán Abad Valenzuela (29) nos ofreció sobre ascaridiosis de las vías biliares y del tubo digestivo, un interesante estudio desde el punto de vista radiológico; y el doctor Miguel Angel Jijón (30) publicó en 1959 su interesante artículo: "Un caso de Ascaridiosis y Hepatitis".

TOXOCARIASIS. El doctor Francisco Pervan (*) en 100 exámenes coprológicos realizados de pacientes niternados en el Hospital de San Juan de Díos die esita ciudad, por el año de 1916, encontró una muestra con huevos de Ascaris canis; en 1924, el doctor Enrique Gallegos Anda (**), en un Informe solbre los trabajos de Laboratorio Clínico de su cátedra, dio a conocer, a los exámenes de las heces, 8 casos con huevos de Ascaris canis. Uno de nosotros (30) en 1963 reportó un caso, en una niña, de toxocariasis por examen coprológico; posteriormente hemos observado en el Laboratorio uncs dos casos más, también en niños; a principios de 1962 fuimos consultados por el doctor César Dávilla Saá, sobre el hallazgo en las heces fecales

Anales de la Universidad Central. IV (57-58): 418, Quito, 1917.

( **) Anales de la Univiensidad Central. XXX (2/8): 104-105, Quito, 1924. de un paciente, de huevos de Ascaris canis.

Por numerosos estudios sobre esta piarasitosis y sobre todo por el trabajo publicado por el doctor P. C. Bea$\operatorname{ver}(* * *)$ sabemos sobre los graves estragos que ocasiona este vemis, especialmiente en los niños, quien sistematizó la sintomatología en los siguientes signos y sítomas: hi pereosinofilia persistente e hiperglobulinemia, hepatomegalia, fiebre recurrente asociada con anorexia, laslitud y disposición a la irritabilidad; no olvidemos que en esta parasitosis existe, además infiltración pumonar, compromiso del sistema nervioso central y endoftalmitis, trastornos todos ellos debido a una toxocariasis por larvas migrans.

\section{RESUIMEN}

El estudio y el conocimiento de las parasitosis hepatobiliames es de trascendental importancia en nuestros países por la frecuencia y gravedad. De ellias, muchos casos son diagnosticados clinicamente gracias a los recursos de laboratorio y a modernos métodos de exploración, pero otros casos son casualmente descubiertos en intervenciones quirúrgicas, y no pocos, en necropsias.

En el Ecuador se han reportado las sliguientes parasitosis hepatoibiliares: amibias s, coccidiosis, balantidiosis, hidatidosis, fascioliasis, paragonimasis, opistorch asis, ascaridiosis y toxocariasis, y como procesos y localizaciones

(***) Bull de la Societé de Patologio Exoti_ que, T. 55 (4): 555-576. 1962. 
hepatobiliares, en las siguientes parasitosis generales: malaría, toxoplasmoslis, kala-azar, enfermedad de Chagas y cisticercosis, aunque esta última, a gual que la paragonimiasis, puede ser de localización sólo hepática.

Son nuevos aportes a la Parasitología y Gastroenterología americana, Ia cocclidiosis hepática, el absceso balantidiano, la paragonimias's y la opistorchiasis.

Se recomienda el estudio, investigación y divulgación de los conocimientos de estas localizaciones y manifestaciones de las parasitosis hepatobil ares en los demás países del Continente.

\section{ABSTRATS}

The study and knowledge of the hepatobiliary parasitosis among our countries is of great importance due to its frequency and risk. Many of them are diagnosed clinically thanks to the help of laboratory methodls as well to the newly exploration methodologies, but in other cases their discovery is casual in surgery or in necropses.

In Ecuador the following parasitic diseases of the liver and billiary ducts had been describer: Amoebiasis, Coccidiosis, Balanthidiosis, Hidatidic cists, Fasciolasis, Paragonimiasis, Opistorchiasis, Ascariasis, Toxocariasis, and as proceses and hepatobiliary localizations in the following general parasitic states: Malaria, Toxoplasmosis, Kalaazar, Chagas disease, Cysticercosis, although in this least as in Paragonimissis the infestation might be only in the liver.
As new the american parasitology and gastroentrolgy are the Hepatic Coccidioisis, the Abcess due to Balantidium, Paragonimiasis and Opistorchiasis.

It is reccomended the study, research and publication of the new foundings of this localizations and pathology in other countries of America in whom they have not been found yet.

\section{BIBLIOGRAFIA}

1 TANCA MARENGO, J. El hígado amibiano (Aspectos Clínicos). Anales de la Sociedad Médico-Quirúrgica del Guayas. XXXVII (7-9); 1627-1636. 1946.

2 MOREIRA, A. Hepatitis amebiana: su clínica y tratamiento. Anales de la Sociedad Médico-Quirúrgica del Guayas, XLIII (10-12); (1-3): 2340-2341. 19511952.

3 BARZALLO, R. Hepatitis amebiana leI del lóbulo izquierdo (Formas tórpidas). Actas del V Congreso Médico Nacional, Tomo I, pp. 342-347. Quito, 1962.

4 CORDERO JARAMILLO, L. Algunas modificaciones encontradas en la amebiasis. Actas del V Congreso Médico Nacional, Tomo I, pp. 335-341. 1962.

5 MOLL, A. Aesculapius in Latin América, p. 206. W. B. Saunders Company, Philadelphia and London, 1944.

6 TROYA, J. M. Contra hechos no hay argumentos. I, pp. 15-16, y II, p. 3. Tipografía de la Escuela de Artes y Oficios, Quito, 1896.

7 DESTRUGe, J. Abscesos hepáticos. Gaceta Médica, I (1): 12-15, 1893.

8 PAREDES, J. E. Forma Pseudo-Neoplástica en la Hepatitis Amebiana. Anales de la Universidad Central, XLII (268): 268 y sgts. 1929. 
9 LEON, L. A. Historia de la Amebiasis hepática en el Ecuador. Inédita.

10 LEN, L. A. Y GUERRERO, F. Coccidiosis hepática. Consideraciones anatomopatológicas y Clínicas. Medicina, Revista Mexicana. XXXIX (813): 45-50. 1959.

Reproducido en: Gaceta Médica, XIV (3): 318-325, Guayaquil, 1959.

11 LEON, L. A. Leishamanias y Leishmaniasis (Especialmente en América), pp. 112-116. Editorial Universitaria, Quito, 1957.

Leishmaniasis visceral. Gaceta Médica, XIII (5): 617-650. Guayaquil, 1957.

12 AUZ, J. L. Absceso Hepático por Balantidium Coli. Memorias de las IX Jornadas Médicas Nacionales. Capítulo de Guayaquil, de PAMA., pp. 63-66. 1967.

13 ESPINOSA TAMAYO, L. Contribution a L'Etude de la Géographie Médicale et des Conditions Hygiéniques de la République de l'Equateur, p. 44. Laussanne, 1917.

14 HEINERT, J. F. Protocolo de autopsia No 135 A del caso de cisticercosis generalizada. Anales de la Saciedad Médico-Quirúrgica diel Guayas, VI (10) 292 $295,1926$.

15 Observación de cisticercosis generalizada. Anales de la Sociedad Médrico-Quirúrgica del Guayas, XII (5): 218 y 222. 1932.

16 ALCIVAR, M. H. Y VALENZUELA, A. Caso de Quiste Hidatídico del Hígado Presentado al Primer Congreso Miédico Ecuatoriano, de 1915. Referencia: Anales de la Sociedlad Médico-Quirúrgica dlel Guayas, IV (4): 160. 1924.

17 DAVILA, L. G. El Quiste hidatídico en el Ecuador. Boletín del Hospital Civil de San Juan de Dios. I (7,8 y 9): 126133. 1926
18 TERAN C., R. Un caso de Equinoccocia del hígado. Rervista del Departamento Médico del Seguro Social Ecuatoriano, I (1): 23-28, 1948.

19 CORDERO JARAMILLO, L. Y CARRERA COBOS, T. Un caso autóctono de Quiste Hidatídico del hígado. Rev. de la Facultad de Ciencias Médicas de la Universidiad de Cuenca, 4 (2): 269-275. 1963.

20 ZEREGA PENDOLA, F. Hidatidosis Al. veolar. Rev. Ecuatorilana de Higiene y Medicina Tropical, 22 (2): 115-117. 1965.

21 ESTEVES EDDERMAN, C., RODRIGUEZ M., J. D. Y. LEONI D. G.,Hidatidosis Hepática: Exéresis de quiste hidatídico unilocular en un paciente autoctono de Guayaquil. Memorias de las X Jornadas Médlicas Nacionales y de las IIIJornadas de Especialidladles. Asociación Médica Panamericana, pp. 179-183.

22 LEON, L. A. Y ABAD, G. Distomatosis hepatobiliar en el niño. Relación de un caso grave observado en la ciudad de Quito, Ecuador. Revista Medicina Mexicana. LI (1105) 169-175; (1106): 185192, y (1107): 209-215. 1971.

23 HEINERT, J. F. Paragonimiasis Pulmonar. Anales de la Sociedard Médlico-Quirúrgica del Guayas, I (13): 43-51. 1922

24 LEON, L. A. Diagnóstico de la Paragonimiasis. Revista KUBA, 13 (1-6) I-4. Habarana, 1955.

Reproducido en: Gaceta Médica, X (6): 903-910. Guayaquil, 1955.

25 Montalvan, J. A. Paragonimiasis en el Ecuador. Estudio Epidemiológico y Clínico. Rev. de la Facultad de Ciencilas Médioas de la Universidad de Guayaqdil, 1 (3): 9-56, 1968.

26 GOMEZ LINCE, L. F., RODRIGUEZ, J. D. Y MONTALVAN, J. A. El Opisthorchis Guayaquilensis. Rev. Ecuatoriana de Higiene y Medicina Tropical, 6 (1-4): 11-14. 1949. 
27 VARAS CALDERON, A. Nuevo Caso de Opistorquiasis en Guayaquil. Gaceta Médica, VI (6): 574, 1951.

28 LEONE DI VANNA, G. Granulomas y Abscesos del Hígado Producido por Ascaris Lumbricoides. Memorias de las Primeras Jornadas Médicas del capítulo de Guayaquil, de la Asociación Médica Panamericana, pp. 390-392, 1957.

29 ABAD VALENZUELA, G. Ascaridiosis de las Vías Biliares y del Tubo Digestivo. Actas del V Congreso Médico Ecuatoriano, T. I: 410-420. 1962.
Reproa'ucido en: Anales de la Sociedad Médico-Quirúrgica del Guayas. 40, Octubre due 1961.

30 JIJON, M. A. Un caso de Ascaridiosis y Hepatitis. Anest. II (2): 7 y sgtes. 1959.

31 LEON, L. A. Toxocariasis en la Infancia. Gaceta Médica, XVIII (2): 143-152. 1963

Dirección de los AA.: Laboratorios de Parasitología y Medicina Tropical, Apartado Postal 457. Quito, Ecuador, S. A. 